\title{
La ciudad y la región: imágenes y realidades revisitadas
}

\author{
Osvaldo Preiss \\ Universidad Nacional del Comahue - Buenos Aires - Argentina \\ ORCID: https://orcid.org/0000-0001-6380-0978 \\ Romina Valeria Schroeder \\ Universidad Nacional del Comahue - Buenos Aires - Argentina \\ ORCID: https://orcid.org/0000-0002-3543-8323
}

\section{Resumen}

En la construcción de un marco teórico que posibilite abordar la relación entre ciudad y región, resulta pertinente analizar si la teoría y la metodología, que se erige como la ortodoxia actual, ha resultado apropiada para el estudio del desarrollo de las regiones, o si las trayectorias urbanas y regionales deben ser abordadas incorporando elementos ausentes, que nos permitan visualizar acabadamente sus dinámicas. Al respecto, consideramos que pretender entender la ciudad descontextualizada de su inserción en regiones y territorios concretos representa una abstracción incompleta, que no sólo fragmenta el análisis de lo urbano sino, además, los procesos de reproducción del espacio en su conjunto. Se propone realizar una revisión de la relación entre ciudad y región, en los fundamentos de las teorías predominantes en la literatura, que constituyen la corriente principal del pensamiento urbano y regional desde los años ochenta, y en las visiones alternativas que destacan la heterogeneidad de las trayectorias de los territorios, especialmente referidas a las contribuciones de autores latinoamericanos.

Palabras clave: Ciudad. Región. Economías de aglomeración. Latinoamérica

\section{Resumo}

\section{A cidade e a região: imagens e realidades revisitadas}

$\mathrm{Na}$ construção de um referencial teórico que possibilite abordar a relação entre cidade e região, é pertinente analisar se a teoria e a metodologia, que se posicionam como ortodoxia vigente, se mostraram adequadas para o estudo do desenvolvimento das regiões, ou se trajetórias urbanas e regionais devem ser abordadas, incorporando elementos ausentes que nos permitam visualizar sua dinâmica. Para nosso conhecimento, pretender entender a cidade descontextualizada de sua inserção em regiões e territórios específicos representa uma abstração incompleta, que não apenas fragmenta a análise do urbano, mas também os processos de reprodução do espaço como um todo. Propõe-se rever a relação entre cidade e região nos fundamentos das teorias predominantes na literatura, que constituem a corrente principal do pensamento urbano e regional desde a década de 1980, e em visões alternativas, que destacam a heterogeneidade do trajetórias dos territórios, especialmente referentes às contribuições de autores latino-americanos.

Palavras-chave: Cidade. Região. Economias de aglomeração.América Latina. 


\title{
The city and the region: images and realities revisited
}

\begin{abstract}
In the construction of a theoretical framework that makes it possible to address the relationship between the city and region, it is pertinent to analyze whether the theory and methodology, that stands as current orthodoxy, has proved appropriate for the study of the development of regions, or if urban and regional trajectories should be addressed incorporating missing elements that allow us to visualize their dynamics. In this sense, we consider that to pretend to recognize the city, decontextualized of its insertion in specific regions and territories represents an incomplete abstraction, which not only fragments the analysis of the urban but also, the processes of reproduction of the space as a whole. The article propose to critically review the relationship between the city and region in the foundations of the predominant theories in the literature, that constitute the mainstream of urban and regional thinking since the 1980s, and in alternative visions that highlight the heterogeneity of the trajectories of the territories, primarily referring to the contributions of Latin American authors.
\end{abstract}

Keywords: City. Region. Agglomeration economies. Latin America.

\section{Introducción}

En el marco de la transformación de los sistemas productivos, devenida de la globalización y la flexibilización post fordista, y junto al cuestionamiento de las políticas tradicionales de incentivos para la transformación de territorios "atrasados", en la década de 1980 tomaron primacía en el campo del desarrollo regional, un conjunto de nuevas elaboraciones teóricas que orientaron el análisis hacia la determinación de las condiciones de competitividad de un conjunto de sistemas locales de producción, que mostraban destacadas trayectorias de crecimiento y competitividad.

En este análisis, la ciudad fue adquiriendo un rol preponderante como actor protagónico de las redes y flujos que sustentaban la globalización y en su condición de ámbito privilegiado de la producción, de la interacción entre actores y generador de ventajas de aglomeración dinámicas, a partir de la incorporación de los factores institucionales, del conocimiento y la innovación, en los que incidía el capital social de las comunidades involucradas.

Aún bajo la hegemonía de estas aproximaciones, que constituyeron una nueva "ortodoxia regionalista", la heterogeneidad de las trayectorias de los espacios subnacionales continuó siendo un rasgo nítido, que mantuvo en cuestión las concepciones de convergencia y las políticas de cohesión que pretendían favorecer el crecimiento de las regiones "rezagadas", dando lugar a que, bajo diferentes perspectivas teórico-metodológicas, la región como objeto de estudio continuara siendo analizada en la tradición histórica de la desigualdad del desarrollo.

Aunque su análisis pueda hacerse en forma separada para ciertos propósitos pues remiten a procesos que, indudablemente, no son similares, ciudad y región son conceptos que siguen manteniendo una relación que hace a su misma conformación y, por ende, su conceptualización no debe derivar en la anulación de la articulación profunda e indisoluble que los une en la realidad (HIERNAUX, 2014). 
No es procedente, entonces, seguir pensando en la ciudad y la región como entidades disjuntas, basadas en la radicalización de la división campo/ciudad o en supuestas dinámicas "modernas y tradicionales", como se sigue afirmando en ciertos paradigmas teóricos que intentan dar lugar a una caracterización contradictoria, en la cual la región queda sometida a la dinámica generativa de la ciudad hasta convertirse, en ciertos casos, en un mero soporte territorial, proveedor de recursos propios del ámbito rural y extractivo y asiento físico de las expansiones urbanas.

En este sentido, no cabe duda de que las ciudades han jugado un papel decisivo como nodos de los procesos de acumulación del capital, desde las fases proteccionistas hasta el neoliberalismo actual; sin embargo, entender la ciudad descontextualizada de su inserción en regiones y territorios, que muestran una particularidad específica en su desarrollo, representa una abstracción incompleta, que no sólo fragmenta el análisis de lo urbano, sino más ampliamente de los procesos de reproducción del espacio en su conjunto.

En este artículo, nos proponemos hacer una revisión de la relación entre ciudad y región en los fundamentos de las teorías predominantes en la literatura, que constituyen la corriente principal del pensamiento urbano y regional desde los años ochenta, y en las visiones alternativas que, sobre las bases de considerar tanto factores geográficos como económicos, analizan el rol de la ciudad en su interacción con la región.

Desde el punto de vista metodológico, se recurre a una revisión de bibliografía específica que, sin pretender ser exhaustiva, permita analizar los rasgos principales de las lecturas sobre la relación entre la ciudad y la región. Se adopta, en primer lugar, un criterio de origen ordenando las referencias según la procedencia de los aportes, en una primera escala internacional para, posteriormente, enfocarnos en las discusiones teóricas que se originaron en el contexto latinoamericano. Esta diferenciación intenta poner de relieve las singularidades de cada ámbito espacial, al tiempo que propone una alternativa a la complejidad de integrar teórica y analíticamente las discusiones de diferentes disciplinas dentro de las Ciencias Sociales. Además, se incorpora un criterio cronológico, agrupando los aportes por décadas o períodos que significaron un quiebre en las condiciones imperantes hasta ese momento.

La primera parte del artículo indaga sobre el concepto de ciudad, su teorización como entidad social y la forma en que estos abordajes son reformulados por las implicaciones de las redes y los flujos, en la configuración urbana actual y en su proyección como fundamento del análisis urbano. Luego, se avanza en el rol de la ciudad enmarcada en los procesos de desarrollo regional, haciéndose una breve mención a los diferentes modelos que exploran las sinergias locales, con especial interés en las ventajas dinámicas de la aglomeración, asociadas a la innovación, y cuestionándose el tratamiento que recibe la región en estas teorías e interpretaciones. Posteriormente, se aborda la relación ciudad-región ya desde la escala latinoamericana, observándose cómo las teorías han sido trasladas al análisis. Por último, se propone reflexionar en torno a las interrelaciones espaciales y los aspectos micro-analíticos de la dinámica territorial, donde las dimensiones socio económica y político institucional, atravesadas por trayectorias históricas y 
marcadas por relaciones de poder diferenciadas, se transforman en aspectos relevantes en el estudio de los procesos territoriales.

\section{La ciudad como imagen disipada y como realidad concreta}

En la introducción del libro "La condición urbana. La ciudad a la hora de la mundialización”, Olivier Mongin (2006, p. 21) elige remitirse a las palabras de Françoise Choay (1996) para comenzar a transitar por el concepto de ciudad, el cual se utiliza “... para designar indistintamente entidades históricas y físicas tan dispares como la ciudad preindustrial, las metrópolis de la era industrial, los conurbanos, las aglomeraciones de diez millones de habitantes, las «ciudades nuevas» y las pequeñas comunas de más de 2.000 habitantes". Esta diversidad de imágenes permite también pensar que a través del tiempo la ciudad amurallada, que marcaba su límite frente al entorno rural, ha dado paso a la diversidad de redes y flujos que extienden las fuerzas de la urbanización sobre gran parte del territorio (SCHROEDER, 2014).

Frente a esta heterogeneidad de la dimensión urbana, las propuestas realizadas desde distintas disciplinas, especialmente desde la Geografía y la Economía, han dado lugar a una variedad de interpretaciones teóricas sobre la forma de delimitar y definir el concepto de ciudad.

Un criterio frecuentemente utilizado ha procedido a definir la ciudad por oposición al campo, empleando diferentes metodologías para su delimitación. De acuerdo con Roca Cladera (2003, p. 20) se utilizan criterios morfológicos, según las características distintivas "del espacio urbanizado y construido con relación al resto del territorio", advirtiendo el autor que esta metodología muestra inconvenientes al intentar explicar los actuales procesos de descentralización urbana (suburbanización, desurbanización). Por otra parte, se utilizan criterios demográficos, empleando la cantidad de habitantes o la densidad poblacional como elemento delimitador. En este sentido, y haciendo referencia al caso de las áreas metropolitanas, se observa que las bajas densidades en el patrón de uso del suelo residencial puede generar conflictos al momento de encontrar un límite entre la ciudad y el campo. Otras delimitaciones incorporan criterios económicos y sociales, en función de la estructura urbana y las formas de vida de la población (producción, intercambio y consumo de bienes y servicios). Finalmente, se pueden utilizar criterios funcionales, vinculados con jerarquía/categorías político-administrativas, así como las relaciones (personas, empresas) en el uso del suelo, de los cuales los más difundidos han sido aquellas delimitaciones basadas en la relación residencia/trabajo (ibíd., p. 22-28). También se encuentran definiciones que combinan dos o más de los criterios anteriores (SCHROEDER, 2014).

Desde la Economía Urbana, Camagni (2016) expresa que la pluralidad de la producción teórica y empírica no ha dado lugar a una teorización aceptada sobre la ciudad como entidad social, sosteniendo la definición de la ciudad como oposición dialéctica al campo, derivada de la división social del trabajo y agotando juntas la totalidad del espacio. En este punto, se retoma el abordaje tradicional de la dinámica económica urbana que alude "al proceso de concentración espacial de la actividad económica a partir de la existencia de economías de aglomeración [...]" (PREISS et al., 2012, p. 20), aunque reconfiguradas a partir de destacar que son las 
ventajas dinámicas de la aglomeración asociadas a la innovación las que marcan las características actuales del comportamiento diferencial de las ciudades.

Sin embargo, y dado que con el transcurso del tiempo no se pueden sostener imágenes fijas de la ciudad, es posible indagar, siguiendo a Castells (2001, p. 208), si en la actualidad la comprensión del fenómeno urbano exige que la visión del "lugar" vinculada a la aglomeración deba ser reemplazada por la de un espacio de redes, o el "espacio de los flujos" basado en la economía del conocimiento y la información, como fundamentos de la producción, la productividad y la competitividad, tanto para empresas como para países, regiones y ciudades. En esta misma línea, el autor entiende que los lugares disipan su significado geográfico, histórico y cultural "...para quedar integrados como lugares funcionales productores de un espacio de flujos que sustituye el espacio de los lugares" (CASTELLS, 1997, p. 424), indicando que el papel que se asigna a la ciudad es doble, como productora de riqueza y como potencial correctora de la desintegración social, derivada de una economía de redes que no incorpora valores sociales (CASTELLS, 2001 apud Schroeder, 2014).

En este línea, Nagy Gábor (2001) advierte que las funcionalidades urbanas no pueden ser ejercidas individualmente sino en forma colectiva; es decir, quien despliega la funcionalidad es la red (sean estás físicas, de comunicación, culturales y de poder, entre las principales) y no la ciudad considerada aisladamente. De esta manera, será necesario analizar el contexto de interdependencia de la ciudad con otros ámbitos, pero especialmente su lugar en una red constituida por diferentes núcleos urbanos.

Sin dejar de reconocer las implicancias del espacio de los flujos en la configuración urbana actual (y en su proyección concreta como fundamento del análisis de lo urbano), una visión crítica de este pensamiento sostiene que el análisis de los procesos de acción a distancia debe comprenderse en su contexto estructural y territorial (Del Cerro Santamaría, 2004). En este sentido, al analizar las ciudades latinoamericanas, Pradilla Cobos (2014) considera que la incorporación de los soportes materiales y los lugares físicos de la ciudad, es decir, su propia realidad material, resultan necesarios para entender la conformación estructural y las prácticas reales de los actores sociales urbanos concretos, sus contradicciones y formas de resolverlas.

Es necesario, entonces, indagar respecto de si la fuerza de la ciudad aún se sigue expresando con plenitud en el mundo de las redes. Siguiendo a Scott (2017, p. 32; traducción propia no textual), "la ciudad existe como un fenómeno social concreto que puede conceptualizarse de manera coherente sobre la base de las dinámicas sociales y políticas que le son propias". El mismo autor, agrega que "el fenómeno de la aglomeración inducida por la división del trabajo y los costos espaciales de la interacción, se ve reforzado por un conjunto de procesos complementarios generados por la creciente masa de la ciudad" (ibíd. p. 22, traducción propia no textual), asociados a las ventajas del crecimiento de la ciudad en términos de las economías de escala, su estructuración a través de los comportamientos y procesos decisorios, que inciden sobre las relaciones sociales y de propiedad, y las formas de gobierno que se establecen para tratar los aspectos de coordinación y manejo colectivos (SCOTT, 2017). 


\section{La ciudad como epicentro del desarrollo}

Alentadas principalmente por el clima de crisis generalizado en las políticas regionales, a partir de mediados de los años 70 y principios de los 80 s del siglo XX, las iniciativas locales de desarrollo económico se hicieron presente para ocupar el lugar de la desacreditada política del desarrollo regional que, inspirada por el pensamiento neokeynesiano que fundamentaba la política económica de la época y recurriendo a las lógicas del modelo de los polos de desarrollo de Perroux (1955), combinaban infraestructura, equipamiento y recursos con importantes ayudas a la inversión pública y privada que se consideraban necesarias para impulsar el desarrollo regional, pero cuya concreción no logró superar los límites del contexto económico e institucional que se pretendía modificar.

Hadjimichalis (2019) sostiene que este cambio de paradigma, en el desarrollo regional de las décadas de 1980 y 1990, llevó a trasladar el foco de atención desde las razones del atraso en regiones periféricas al estudio de casos de regiones exitosas, introduciendo lo local como escala de análisis. Es así que, en las últimas cuatro décadas, economistas regionales, geógrafos y planificadores urbano regionales han avanzado en el análisis de los procesos de desarrollo local como objeto de estudio compartido por la Economía y la Geografía, destacando los efectos de las economías de aglomeración en la concentración de ciertas actividades económicas en la ciudad. Según Trullén (2006),

\footnotetext{
Se trata de un amplio conjunto de economías externas que dan ventaja a la concentración espacial de la actividad y de la población y que se clasifican tradicionalmente en tres subconjuntos: economías internas a la empresa (que definen las áreas de mercado de las empresas), economías externas a la empresa pero internas a la industria (o economías de localización), y economías externas a la empresa y a la industria (o economías de urbanización).
}

Dos concepciones estuvieron presente en los orígenes de la nueva aproximación: la teoría de cluster y los distritos industriales (DI). Si Porter incorporó la "proximidad geográfica" a su concepción de interacción de firmas de distinto tamaño, Becattini definió de inicio a los distritos industriales como "entidades socio territoriales" en las que tenían un rol protagónico las pequeñas y medianas empresas. En este sentido, Sforzi (2009), reivindica a la teoría de los DI como heredera de la Economía Regional, por contraposición al origen de la visión del cluster, que derivaría de las teorías de la localización industrial, distinción que no siempre es atendida suficientemente.

Siendo que estas nuevas teorías derivaron del interés en posicionar la industrialización localizada como objeto de estudio, el centro de atención se desplazó desde las economías de aglomeración estáticas a las condiciones de sinergia e innovación que genera la interacción de actores geográficamente próximos, incluyendo su relación con la sociedad local.

En líneas generales, se postula que las externalidades de aglomeración estática se convierten en dinámicas, generando no solo una reducción de costos y una expansión de los ingresos sino una limitante a la incertidumbre dinámica, típica de los procesos de innovación, y una expansión de las ganancias schumpeterianas a través de la novedad, el emprendimiento efectivo y la innovación (CAMAGNI, 1991). 
De allí se deriva la idea que, si se pretende que el crecimiento urbano sea desarrollo, cambio estructural e innovación, existiría un acuerdo suficiente de que la ciudad debe analizarse como locus, donde la mayor parte de la producción industrial, los trabajadores calificados y los altos salarios tienden a aglomerarse, en espacios y tiempos concretos. La ciudad concentra el crecimiento económico, favorecido por la proximidad física entre agentes, lo que facilita la comunicación y crea un ambiente que estimula la interacción y el flujo de ideas, y donde las actividades intensivas en conocimiento resultan fundamentales para el desenvolvimiento económico (IAMMARINO, 2005; ASCANI et al., 2012).

Entre las nuevas interpretaciones de la ciudad, autores como Camagni (2016), Cusinato y Philippopoulos-Mihalopoulos (2016) hablan de "milieu cognitivo" haciendo hincapié en el análisis de los aspectos simbólicos del contexto urbano resaltando, por ejemplo, la importancia de los espacios físicos públicos, simbólicamente reconocidos y apropiados por una comunidad local, como una forma de construcción identitaria y generadores de disfrute, emoción y una atmósfera de relación, incluso afectiva. De esta manera, se trabaja con una idea de "ciudad generativa" en la cual la creatividad, el conocimiento e innovación socio cultural, toman un rol protagónico apoyada en las cualidades y percepciones antes señaladas.

Por otro lado pero en relación con lo anterior, la centralidad que asumió el análisis de la ciudad nos conduce a preguntarnos respecto del lugar que ocupa la región en estas teorías. Partiendo de la constatación que las ciudades que concentran el crecimiento económico se localizan en un conjunto de regiones en las cuales las interacciones entre los actores locales y un marco institucional favorable configuran una capacidad de innovación ligada a contextos específicos (IAMMARINO, 2005; ASCANI et al., 2012), se postuló que el desarrollo local resulta pasible de ser estimulado mediante una política regional que dinamice las competencias de las estructuras socio institucionales y culturales.

Así, y tomando como referencia los distritos italianos en la región de Emilia Romana, las políticas regionales han evolucionado desde la creación de infraestructura (cuyo típico exponente fueron los parques industriales municipales), entre las década de 1950 a 1970, a la institucionalización de agencias que permitieran reparar las fallas de mercado en cuanto a las posibilidades de acceso a la información a las redes de pequeñas y medianas empresas de los DI, en la década de 1980, modificándose en los años 90 s hacia un sistema de innovación regional que permitiera favorecer la difusión, el intercambio y la creación del conocimiento para mejorar la capacidad innovativa de los actores (BIANCHI et al., 2018).

Sin embargo, el proceso de concentración empresarial, mediante adquisiciones y fusiones, que cobró fuerza en los años noventa, la creciente integración de cadenas globales de producción y los efectos de la crisis mundial a partir del año 2008, han evidenciado la importancia de los factores del orden económico internacional y la reacción diferenciada de las regiones que se ven impactadas por la aparición de empresas trasnacionales.

La dinámica del orden económico global llevó a reconocer que los procesos de penetración del capital desarticulan progresivamente las fortalezas identificadas en las regiones consideradas como "exitosas", especialmente, cuando se trata de sistemas de pequeñas y medianas empresas. Esto ha trasladado el foco de los 
estudios en los cluster y DI hacia sus diferentes procesos evolutivos y la ampliación de los límites geográficos de sus interacciones en una nueva división territorial del trabajo (DE MARCHI et al., 2018).

\section{Teorías latinoamericanas: ciudad, región y territorio}

Desde una mirada latinoamericana, Hiernaux (2014) plantea la existencia de dos momentos importantes en el estudio de la relación entre ciudad y región. Por un lado y desde una perspectiva sociológica, el que remite a la teoría de la modernización desarrollada por Gino Germani donde se cuestiona el crecimiento de las ciudades "en un contexto de articulación con mercados internacionales, sin efectos en el resto del país (lo que posteriormente Cardoso y Faletto caracterizarán como condiciones de "enclave")" (ibíd., p. 44). Este planteamiento - muy discutido por cierto desde los sectores críticos hasta bien entrada la década de 1980 - se basa en la dualidad campo/ciudad y coloca a la región como un espacio relegado en el cual la ciudad actúa como un mecanismo posible de integración de la población a la modernidad. Asimismo, autores como Duhau López (2014), destacan la influencia en los estudios latinoamericanos de los años setenta de la nueva sociología urbana francesa - de corte neomarxista y de inspiración lefebvriana y althusseriana - cuyo objeto de estudio principal refiere al papel de la ciudad en el proceso de acumulación y reproducción de la fuerza de trabajo.

En gran medida, los fundamentos de las relaciones que se postulaban entre ciudad y desarrollo, estaban basados en teorías generales entonces enfrentadas en un intenso debate en torno a los planteamientos particulares sobre dichas temáticas (RAMÍREZ VELÁZQUEZ y PRADILLA Cobos, 2014). Si bien, en América Latina, constituía una discusión intelectual entre "investigadores militantes con diversas opciones políticas", en lo que refiere particularmente al análisis urbano, se limitaba a teorizaciones con una fuerte impronta de las escuelas de sociología estadounidense y francesa (ibíd., p. 11).

La segunda propuesta giró en torno al "desarrollo polarizado" elaborada por el economista François Perroux, que parte de la premisa que "el crecimiento no es ubicuo, se concentra en ciertos nodos de la economía donde aparecen factores de innovación [y así generar] "efectos multiplicadores” (Duhau López, 2014, p. 44-45) en la región de influencia. Esta hipótesis - trabajada como "polos de desarrollo regional” - será de interés para la disciplina geográfica en una época (1950) donde los enfoques históricos-descriptivos de la Geografía Regional Francesa se encontraban muy cuestionados y las teorías económicas ganaban peso en el estudio del espacio. En tal sentido, como expresa Hiernaux (2014, p. 45), “... varios autores latinoamericanos se dejaron convertir [...] en un momento en que se carecía de propuestas para el desarrollo regional en América Latina y ya se empezaba a percibir el riesgo generado por la fuerte concentración urbana que llegaba a ahogar las regiones". Sin embargo, la propuesta despertó reacciones en contra, entre ellas el análisis crítico que haría José Luis Coraggio de las políticas regionales sustentadas en esta teoría. Según el autor (apud HIERNAUX, 2014) los polos no difunden en forma adecuada ciertos factores de modernidad y de transformación sino que, además, propician un aumento de la penetración de la economía capitalista en el 
entorno regional y generan desequilibrios en las ya endebles economías regionales en donde se implementan.

Las opciones teóricas cambiaron en los años ochenta en un contexto general de desplazamiento paulatino del modelo de sustitución de importaciones hacia políticas orientadas al mercado desregulado, que van adoptando los diferentes países latinoamericanos, y que influyen significativamente en los problemas de investigación y en los variados abordajes adoptados dentro del ámbito académico.

En los últimos años, se ha observado una creciente diversificación de los temas y los enfoques teóricos de la investigación urbana, en la cual Duhau López (2014, p. 22-23) destaca tres líneas o ejes principales: (1) el patrón de urbanización e impacto de la globalización en la reestructuración económica y espacial, y las formas de producción de las ciudades, particularmente influenciada por la teoría de las ciudades globales; (2) la reestructuración social de las ciudades, con énfasis en la pobreza urbana y la polarización social y (3) los vínculos y el rol que asumen los espacios locales, en términos de gestión gubernamental, ciudadanía, democracia y la articulación de diferentes actores sociales.

En el plano académico, esto significó una mayor concentración en el estudio de la ciudad que, particularmente, recayó sobre la dinámica de las grandes metrópolis, mientras que disminuyó sensiblemente el estudio de su relación con la región y, en muchos sentidos, se produjo un quiebre en que la región quedó bajo la mirada del desarrollo rural, aunque hubo esfuerzos de conectarla en su vinculación con la ciudad.

Particularmente, Hiernaux (2014) al realizar una apreciación crítica de los estudios de la relación ciudad-región, destaca dos grandes líneas de trabajo en las últimas décadas, por un lado, la noción de "sistema de ciudades" que, de alguna manera, retoma el planteo clásico de la teoría de los lugares centrales, que entiende a la ciudad como motor del espacio circundante, además de establecer que "los flujos más importantes entre ambos espacios son los que marcan la dependencia, la subordinación de la región a la ciudad central y, a menor escala, la subordinación de áreas "consumidoras" al centro productor". En este sentido y como considera el autor, "Los estudios sobre sistemas de ciudades en buena medida también se derivan de la teoría de los polos de desarrollo, y generan así una suerte de hibridación de varias corrientes de la economía espacial” (ibíd., p. 49). El énfasis está puesto en las dimensiones culturales de las regiones, reinterpretando algunos componentes del DI, que trabaja con la idea de una suerte de "personalidad regional"

Otra parte de los estudios giran en torno a la desindustrialización/reindustrialización de los países latinoamericanos y la incorporación de la escala regional y cómo ésta se inserta en los procesos globales. En este sentido, Hiernaux (2014) observa un cambio significativo en el patrón de relaciones entre ciudad y región: "mientras que las primeras se desindustrializan y se convierten en economías terciarizadas, las segundas acogen nuevas industrias las cuales generan el crecimiento de ciudades medias que no pasaban generalmente de ser centros regionales menores" (ibíd., p. 51). Aunque el autor aclara que las grandes ciudades no pierden el control del sistema regional sino que lo ejercen, principalmente, a través de los flujos financieros y “... sobre todo, por el hecho de 
concentrar las principales funciones de mando y las sedes de las plantas industriales que se dispersaron a lo largo de las economías regionales" (ibíd.)

Esto marca una tendencia en los estudios en Latinoamérica, en la que el cuerpo de trabajo, impulsado fundamentalmente por el éxito y el dinamismo de ciertas economías regionales de alto perfil en Europa occidental y América del Norte, se unió en torno a una comprensión de la forma específica de las relaciones entre empresas y las condiciones institucionales y sociales que los caracterizaban, para constituirse en una suerte de marco de referencia para la mayor parte de los investigadores, donde la diversidad se unificaba en el concepto de desarrollo territorial.

Autores con gran influencia en el ámbito latinoamericano (MAZUREK, 2012; MENDÉZ, 2006a; ALBUQUERQUE, 1999) comienzan a poner atención en los aspectos micro-analíticos de la actividad productiva, dejando de interpretar el espacio como un mero soporte físico de actividades económicas para pasar a comprender el territorio y su consecuente transformación en el tiempo; esto es, considerar las formas en que los diferentes actores sociales se relacionan; sus organizaciones concretas y sus técnicas productivas; la relación con el medio y la movilización social y cultural (SCHROEDER, 2014; SCHROEDER y NOYA, 2017); revalorizando el propio concepto de cluster y DI.

De esta manera, el territorio asume un rol protagónico donde “...la economía del conocimiento, los procesos de innovación, la calidad, la logística, y los procesos de aprendizaje aumentan su importancia en las performances competitivas" (BOSCHERINI y POMA, 2000, p. 16).

En este contexto, si la innovación y la integración a la red de flujos define la competitividad de los sistemas localizados de producción basados en la fortaleza de los espacios urbanos, el territorio es definido como un conjunto de instituciones, organizaciones y sistemas de interacción de actores que brindan condiciones externas e internas a los agrupamientos de empresas y expresan la capacidad de la región para generar factores institucionales, sociales y de conocimiento que generen una acción sistémica orientada al desarrollo. En este sentido, tuvieron mayor consideración en Latinoamérica las teorías respecto de sistemas territoriales de innovación (Méndez, 2006a; 2006b); sin embargo, este enfoque presenta una debilidad en cuanto a las consideraciones de las relaciones de poder diferenciadas y los límites de los procesos que operan en el territorio frente a los presupuestos exigidos.

El intento de reinterpretación del DI hacia un sistema institucional territorial que permitiera superar estas limitaciones resulta particularmente interesante, dado que avanza sobre la visión tradicional incorporando al territorio como sujeto en la producción de conocimientos “...en el que, y con el cual, el tejido productivo interactúa” (BOSCHERINI y POMA, 2000a, p. 17) y, al mismo tiempo, compite con otros territorios. De esta manera, se relacionan la dimensión socio económica e institucional, al indagar acerca del rol que cumplen agentes locales y organizaciones territoriales (cámaras empresariales, gremios, universidades, agencias de desarrollo, etc.) ya no como una externalidad sino como participantes directos del proceso competitivo (ibíd.). 
Particularmente para el caso latinoamericano, la posibilidad de estructurar un sistema institucional en realidades diferentes, a las que originalmente despertaron el interés de los académicos del DI italiano, presentó limitaciones en cuanto a su alcance. Al respecto Boscherini y Poma (2000b, p. 29), al analizar el sector industrial argentino, hablan de la "fuerte concentración territorial en los centros urbanos" y de la escasa o nula articulación con la región; una realidad socio económica altamente condicionada por las "especificidades e idiosincrasias" locales, donde predomina la tutela del Estado, la mirada cortoplacista, una escasa predisposición y falta de confianza en las acciones colectivas y en las relaciones entre los diferentes actores sociales. Referido a esto último, los autores han sido tajantes al afirmar que,

[...] las debilidades de la sociedad civil y el insuficiente grado de desarrollo del sistema político, tanto en el ámbito nacional como en el local, no han posibilitado la generación de un sistema institucional capaz de estimular y apoyar la creación de sistemas productivos locales. La ausencia de relaciones entre la dimensión pública y privada, la estructura "jerárquica" de las relaciones Estado-sociedad civil y el predominio de comportamientos individualistas no han generado una trama institucional flexible y susceptible de representar un estímulo para el desarrollo del sector productivo (ibíd., 31).

En síntesis, el énfasis en las sinergías y los procesos de concertación, como medios para el desarrollo de los territorios, desconoció las condiciones diferenciales de las relaciones entre los actores y la carencia de un poder regulatorio de las instituciones públicas, desdibujando así el mismo concepto de territorio, debilitando tanto las propuestas teóricas como prácticas, con lo cual sus análisis y recomendaciones terminaron frecuentemente en diagnósticos que poco útiles fueron en la interpretación de las realidades particulares y los instrumentos de política propuestos.

\section{Consideraciones finales}

El análisis realizado pone de relieve las controversias y dificultades metodológicas que surgen al teorizar sobre la relación entre la ciudad y la región. Monasterio y Cavalcante (2011, p. 44; traducción propia no textual) expresan al respecto que “... no existe un enfoque teórico único en la economía regional. Lo que hay es una diversidad de puntos de vista que intentan capturar toda la complejidad de los fenómenos económicos en el espacio".

Como plantea Bertoncello (2007, p. 7-8), en los tiempos actuales, “... la crítica a los grandes discursos parece habernos acostumbrado a la circulación y aceptación acrítica de términos y propuestas cuyos contenidos y fundamentos no siempre están claros pero que son aceptados sin mayor discusión...". En este sentido, se observan claramente las limitaciones que presentan las propuestas teóricas cuando se pretende adoptarlas para el análisis de las realidades latinoamericanas sin el tamiz de la reflexión crítica, con escaso o nulo cuestionamiento a su validez, esto es, poner en tensión el significado y los reales alcances de dichas propuestas.

Lo anterior no es exclusivo de los ámbitos académicos, donde se suelen construir, en algunos casos, "marcos teóricos" sin preguntarse cómo se concretan 
en lo empírico o sobre qué contextos (sociales, políticos, institucionales) teorizan los autores citados sino que también se extrapola a la esfera política, más precisamente, a la gestión pública, aportando a un sinsentido de conceptos y prácticas que, en muchos casos, termina trasladándose a las acciones de los propios actores territoriales, agotando esfuerzos y expectativas en los participantes convocados a integrarse a programas y proyectos de organismos nacionales e internacionales.

A pesar del predominio que han adquirido estas aproximaciones en la academia y la política en América Latina, una corriente de autores ha continuado la tradición de buscar explicaciones que, dentro del contexto del proceso multisecular de la mundialización del capital, respondan por las asimetrías causadas por el desarrollo desigual de las formaciones sociales, las diferencias que producen las relaciones de dominación cada vez más profundas entre las naciones, las crecientes desigualdades sociales, los procesos de segregación y exclusión socio territorial, entre los aspectos principales (RAMÍREZ VELÁZQUEZ y PRADILLA Cobos, 2014).

En relación con lo anterior, la tarea de construir un aporte al estudio del territorio no puede prescindir de la socialización y discusión colectiva de estas ideas, tendiendo a encontrar elementos comunes que articulen debates y diferencias y que posibiliten la revisión de la producción académica de otras latitudes pero, sobre todo, la reflexión constante acerca de la realidad latinoamericana en diversas escalas.

El hecho que resulta relevante, entonces, es determinar si la teoría y la metodología que se erige como la ortodoxia actual resulta apropiada para el estudio del desarrollo de las regiones, inclusive en los casos de las regiones exitosas, o si los hechos que marcan la heterogeneidad en el desarrollo regional debe ser abordada incorporando elementos ausentes en el análisis que no permiten visualizar acabadamente las dinámicas regionales.

Específicamente, en el caso de nuestro continente, la ciudad presenta un intenso flujo de relaciones con su entorno inmediato; flujo bidireccional en el cual la región requiere servicios y la ciudad demanda actividades de su hinterland que impulsen su abastecimiento y actividad económica. No es posible pensar que el centrarse exclusivamente en las economías de aglomeración, los procesos de aprendizaje, las redes de ciudades, u otros, puedan constituir una explicación monocausal suficiente para expresar las trayectorias en ningún nivel de recorte espacial.

La construcción de una propuesta alternativa, que permita reelaborar la rica tradición del análisis regional latinoamericano, en una aproximación critica a la comprensión de la evolución de las regiones pasa, como plantea Brandão (2004, p. 59; traducción propia, no textual), por “... el reconocimiento de la imposibilidad de una teoría general y abstracta (lista y acabada) de la problemática regional y urbana, trayendo tal objeto al lugar de donde él nunca podría haber salido: ámbito de la discusión estructural del proceso de desarrollo".

Referido a esto último, deben cobrar relevancia los procesos de división espacial del trabajo, la deslocalización del capital, la constitución de redes de conocimiento diferenciadas, las funciones de las ciudades, las conformaciones de las estructuras de distribución del ingreso y los mercados laborales, las articulaciones entre los gobiernos y actores con diferentes capacidades, la dinámica 
de la inversión, entre los principales. Por ello, es necesario siempre mantener la conexión de los espacios (ciudad, región, sistema territorial) con los procesos globales y locales. De allí que un estudio centrado en flujos y redes formará parte, pero no será tomado en forma exclusiva, como uno de los factores a incorporar en el análisis, ya que cuando analizamos el territorio “... debemos tener en cuenta la interdependencia y la inseparabilidad entre la materialidad, que incluye la naturaleza, y su uso, que incluye la acción humana, es decir, el trabajo y la política" (SANTOS y SILVEIRA, 2001, p. 247).

En tal sentido, la investigación sobre las ciudades y regiones debe combinar las variadas dimensiones que configuran su trayectoria. Para enfrentar esta tensión conceptual que expresa, una vez más, la búsqueda de una aproximación teórica (nunca acabada) a la relación entre ciudad y región, resultará necesario integrar al cuerpo de análisis la reflexión crítica sobre la reproducción de los procesos económicos, los condicionantes históricos y/o geográficos, las limitaciones políticas y/o institucionales, factores que resultan centrales al momento de indagar sobre el funcionamiento económico-territorial (PREISS, 2014).

\section{REFERENCIAS}

ALBURQUERQUE, F. Identidad y territorio. En: ELGUE, M. (Comp.). Globalización, desarrollo local y redes asociativas. Buenos Aires: Corregidor, 1999, p. 33-49.

ASCANI, A.; CRESCENZI, R. y IAMMARINO, S. Regional Economic Development: A review. WP 1/03 Search Working Paper, 2012. Disponible en:

<http://www.dphu.org/uploads/attachements/books/books_3276_0.pdf> Acceso: 20 ago.2019.

BECATTINI, G. Dal settore 'industriale al =distretto' industriale. Alcune considerazioni sull'unità d'indagine dell'economia industriale. Rivista di economía e politica industriale, v. 5, n. 1, p. 7-21, 1979.

BERTONCELLO, R. Prólogo. En: MANZANAL, M.; ARZENO, M. y NUSSBAUMER, B. Territorios en construcción: actores, tramas y gobiernos, entre la cooperación y el conflicto. Buenos Aires: Fundación Centro Integral Comunicación, Cultura y Sociedad - CICCUS, p. 7-14, 2007.

BIANCHI, P.; GIARDINO, R.; LABORY, S.; RINALDI, A. y SOLINAS, G. Regional Resilience: lessons from a historical analysis of the Emilia Romagna Region in Italy. 2018. Disponible en: http://wehc2018.org/wp-content/uploads/2018/07/Bianchi-et-alWEHC-paper_Final.pdf Acceso: 19 ago.2019.

BOSCHERINI, F. y POMA, L. Introducción. En: BOSCHERINI, F. y POMA, L. (Comp.). Territorio, conocimiento y competitividad de las empresas: el rol de las instituciones en el espacio global. Madrid/Buenos Aires: Miño y Dávila Editores, p. 15-21, $2000 a$. 
BOSCHERINI, F. y POMA, L. Más allá de los Distritos Industriales: el nuevo concepto de territorio en el marco de la economía global. En: BOSCHERINI, F. y POMA, L. (Comp.). Territorio, conocimiento y competitividad de las empresas: el rol de las instituciones en el espacio global. Madrid/Buenos Aires: Miño y Dávila Editores, 200ob, p. 23-38.

BRANDAO, C. A busca da utopia do planejamento regional. Revista Paranaense de Desenvolvimento, n.120, p.17-37, ene./jun., 2011.

CAMAGNI, R. (Ed.). Innovation Networks: Spatial Perspectives. London/New York: GREMI/Belhaven Press, 1991.

CAMAGNI, R. Afterthoughts on urban economic theory and its focus. Investigaciones Regionales - Journal of Regional Research, 36, p. 87-105, 2016.

CASTELLS, M. La ciudad de la nueva economía. Papeles de Población, v. 27, p. 207221, ene./mar., 2001.

CASTELLS, M. El surgimiento de la sociedad de redes. Madrid: Alianza, 1997.

CUSINATO, A., y PHILIPPOPOULOS-MIHALOPOULOS, A. (Eds.) Knowledge-creating milieus in Europe: firms, cities, territories. Berlin: Springer, 2016.

DEL CERRO SANTAMARÍA, G. Ciudades y globalización: un enfoque teórico. Revista Española de Sociología, n. 4, p. 199-218, 2004.

DE MARCHI, C; DI MARIA, E. y GEREFFI, G. Industrial districts, clusters and global value chains: toward an integrated framework. En: DE MARCHI, C; DI MARIA, E. Y GEREFI, G. Local Clusters in Global Value Chains. London: Routledge, p. 1-19, 2018.

DUHAU LÓPEZ, E. La investigación urbana y las metrópolis latinoamericanas. En: RAMÍREZ VELÁZQUEZ, B. y PRADILLA COBOS, E. (Comp.). Teorías sobre la ciudad en América Latina. Universidad Autónoma Metropolitana; México D.F., p. 15-39, 2014 .

GÁBOR, N. Knowledge based development: opportunities for medium-sized cities in Hungary. European Urban and Regional Studies, v. 8, p. 329-339, 2001.

HADJIMICHALIS, C. "New" Questions of Peripherality in Europe or How Neoliberal Austerity Contradicts Socio Spatial Cohesion. En: LANG, T. y GÖRMAR, F. (Eds.).

Regional and Local Development in Times of Polarisation: Re-thinking Spatial Policies in Europe, University of Leipzig: Palgrave Macmillan, p. 61-78, 2019.

HIERNAUX NICOLAS, D. Las ciudades y las regiones. En: RAMÍREZ VELÁZQUEZ, B. y PRADILLA COBOS, E. (Comp.) Teorías sobre la ciudad en América Latina. México D.F.: Universidad Autónoma Metropolitana, p. 40-56, 2014. 
IANMMARINO, S. An evolutionary integrated view of Regional Systems of Innovation: Concepts, measures and historical perspectives. European Planning Studies, 13, p. 497-519, 2005.

MAZUREK, H. 2012. Espacio y Territorio. Instrumentos metodológicos de investigación social. 2 ed. La Paz: Fundación PIEB. Disponible en:

https://www.researchgate.net/publication/259779225_Mazurek_H_2012_Espacio_y Territorio_Instrumentos_metodologicos_de_investigacion_social_Segunda_edicion LL_Paz_Fundacion_PIEB_206p Acceso: 19 ago.2019.

MÉNDEZ, R. La construcción de redes locales y los procesos de innovación como estrategia de Desarrollo Rural. Problemas del Desarrollo. Revista Latinoamericana de Economía, v. 37, n.147, p. 217-240, 2006a.

MÉNDEZ, R. Del distrito industrial al desarrollo territorial: estrategias de innovación en ciudades intermedias. Ponencia presentada en IX Seminario Internacional de la Red Iberoamericana de Investigadores sobre Globalización y Territorio. Universidad Nacional del Sur, Bahía Blanca, Argentina, 2006b.

MONASTERIO, L. y CAVALCANTE, L. Fundamentos do pensamento econômico regional. En: DE OLIVEIRA CRUZ, B (et al.) (Org.) Economia regional e urbana: teorias e métodos com ênfase no Brasil. Brasília: Ipea, p. 43-78, 2011.

MONGIN, O. La condición urbana: la ciudad a la hora de la mundialización. $1^{\text {a }}$ ed. Buenos Aires: Paidós, 2006.

PERROUX, F. Note sur la notion de «pôle de croissance». Economie Appliquée, n.8, 1955. Regional Economics, (pp. 93-103). Republicado y traducido por MC KEE, D. L.; DEAN, R. D. y LEAHY W. H. (Eds.). New York: The Free Press, 1970.

PORTER, M. The competitive advantages of nations. London: Macmillan, 1990.

PREISS, O.; GORENSTEIN, S.; HERNÁNDEZ, J.; LANDRISCINI, G.; NAPAL, M.; URRIZA, G. y OLEA, M. Principales discusiones en torno a la dinámica de las ciudades intermedias. En: GORENSTEIN, S.; HERNÁNDEZ, J. y LANDRISCINI, G. (Comp.)

Economía urbana y ciudades intermedias: trayectorias pampeanas y norpatagónicas. Buenos Aires: CICCUS, p. 19-46, 2012.

RAMÍREZ VELÁZQUEZ, B. y PRADILLA COBOS, E. Presentación. En: RAMÍREZ VELÁZQUEZ, B. y PRADILLA COBOS, E. (Comp.). Teorías sobre la ciudad en América Latina. Universidad Autónoma Metropolitana; México D.F., p. 11-14, 2014.

ROCA CLADERA, J. La delimitación de la ciudad: ¿una cuestión imposible? Ciudad y Territorio. Estudios Territoriales, n. 135, p. 17-36, 2003.

SANTOS, M. y SILVEIRA, M. L. O Brasil: território e sociedade no início do século XXI. Río de Janeiro: Record, 2001. 
SCHROEDER, R. Potencial turístico recreativo en el continuo urbano rural. La ciudad de Bahía Blanca como centro de distribución regional. 2014. Tesis Doctoral (Programa de Posgrado en Geografía) - Universidad Nacional del Sur, Bahía Blanca, 2014.

SCHROEDER, R. y NOYA, N. Añelo: convergencia y conflicto en la producción social del espacio. Una aproximación desde los actores sociales. En: GARRIZ, E. (Comp.) Dinámica urbana y el proceso de crecimiento en contextos diferenciados.

Universidad Nacional del Sur; Bahía Blanca: EdiUNS, p. 269-300, 2017.

SCOTT, A. The Constitution of the City: Economy, Society, and Urbanization in the Capitalist Era. University of California: Palgrave Macmillan, 2017.

SFORZI, F. The empirical evidence of industrial districts in Italy. En BECATTINI, G.; BELLANDI, M.; DE PROPRIS, L. (Eds). A Handbook of Industrial Districts.

Cheltenham, UK: Edward Elgar, p. 327-342, 2009.

STORPER, M. y SCOTT, A. J. The geographical foundations and social regulation of flexible production complexes. En: WOLCH, J. y DEAR, M. (Eds.). The Power of Geography. London: Allen y Unwin, 1988.

TRULLÉN, J. Producción y espacio urbano: algunos problemas económicos. En TARROJA, A. y CAMAGNI, R. La nueva cultura del territorio. Diputació de Barcelona, 2006.

Osvaldo Preiss. Universidad Nacional del Comahue. Facultad de Economía y Administración.

Romina Valeria Schroeder. Instituto Patagónico de Estudios de Humanidades y Ciencias Sociales (IPEHCS - CONICET). Dpto. de Geografía, Facultad de Humanidades, Universidad Nacional del Comahue.

Como citar: PREISS, Osvaldo; SCHROEDER, Romina Valeria. La ciudad y la región: imágenes y realidades revisitadas. Redes (St. Cruz Sul, Online), Santa Cruz do Sul, v. 25, n. 2, p. 635651, maio 2020. ISSN 1982-6745. Disponível em: https://online.unisc.br/seer/index.php/redes/article/view/14033 Acesso em: 15 maio 2020. doi: https://doi.org/10.17058/redes.v25i2.14033 


\section{CONTRIBUIÇÃO DE CADA AUTOR}

a. Fundamentação teórico-conceitual e problematização: Osvaldo Preiss e Romina Valeria Schroeder.

b. Pesquisa de dados e análise estatística: Osvaldo Preiss e Romina Valeria Schroeder.

c. Elaboração de figuras e tabelas: Osvaldo Preiss e Romina Valeria Schroeder.

d. Fotos: Não se aplica.

e. Elaboração e redação do texto: Osvaldo Preiss e Romina Valeria Schroeder.

f. Seleção das referências bibliográficas: Osvaldo Preiss e Romina Valeria Schroeder.

Proyecto: Complejos Productivos y Economía Urbana en la Norpatagonia (E114) y Proyecto: Nordpatagonia: nuevas lógicas y continuidades en el uso del territorio (H167), ambos financiado por la Universidad Nacional del Comahue. 